J. Dairy Sci. 95:7225-7235

http://dx.doi.org/10.3168/jds.2012-5406

(C) American Dairy Science Association ${ }^{\circledR}, 2012$.

\title{
Validation of mid-infrared spectrometry in milk for predicting body energy status in Holstein-Friesian cows
}

\author{
S. McParland, ${ }^{* 1}$ G. Banos,† B. McCarthy, ${ }^{*}$ E. Lewis, ${ }^{*}$ M. P. Coffey,‡ B. O’Neill, ${ }^{\star}$ M. O’Donovan, ${ }^{*}$ E. Wall,‡ \\ and D. P. Berry* \\ *Animal and Grassland Research and Innovation Centre, Teagasc, Moorepark, Fermoy, Co. Cork, Ireland \\ †Department of Animal Production, Faculty of Veterinary Medicine, Aristotle University of Thessaloniki, Greece \\ $\ddagger$ Animal and Veterinary Sciences Research Group, SAC, Easter Bush, Midlothian, EH25 9RG, United Kingdom
}

\section{ABSTRACT}

Cow energy balance is known to be associated with cow health and fertility; therefore, routine access to data on energy balance can be useful in both management and breeding decisions to improve cow performance. The objective of this study was to determine if individual cow milk mid-infrared spectra (MIR) could be useful to predict cow energy balance across contrasting production systems. Direct energy balance was calculated as the differential between energy intake and energy output in milk and maintenance (maintenance was predicted using body weight). Body energy content was calculated from (change in) body weight and body condition score. Following editing, 2,992 morning, 2,742 midday, and 2,989 evening milk MIR records from 564 lactations on 337 Scottish cows, managed in a confinement system on 1 of 2 diets, were available. An additional 844 morning and 820 evening milk spectral records from 338 lactations on 244 Irish cows offered a predominantly grazed grass diet were also available. Equations were developed to predict body energy status using the milk spectral data and milk yield as predictor variables. Several different approaches were used to test the robustness of the equations calibrated in one data set and validated in another. The analyses clearly showed that the variation in the validation data set must be represented in the calibration data set. The accuracy (i.e., square root of the coefficient of multiple determinations) of predicting, from MIR, direct energy balance, body energy content, and energy intake was 0.47 to $0.69,0.51$ to 0.56 , and 0.76 to 0.80 , respectively. This highlights the ability of milk MIR to predict body energy balance, energy content, and energy intake with reasonable accuracy. Very high accuracy, however, was not expected, given the likely random errors in the calculation of these energy status traits using field data.

Received February 2, 2012.

Accepted August 1, 2012.

${ }^{1}$ Corresponding author: sinead.mcparland@teagasc.ie
Key words: dairy, energy balance, infrared spectrum, prediction

\section{INTRODUCTION}

In early lactation in particular, dairy cattle, like all other mammals, mobilize body fat reserves to meet the energy demand of, among other processes, lactogenesis. When energy intake is less than energy output, the animal is in negative energy balance. Negative energy balance is a particular problem in high-yielding dairy cows in early lactation because they are unable to ingest sufficient dietary energy to meet the energy demanded to fulfill the milk production level of which they are genetically capable. Consequently, health and fertility are compromised (Dillon et al., 2006).

Energy balance or a suitable measure of body energy status would be useful in multi-trait genetic evaluations as a heritable indicator of genetic merit for health and fertility in dairy cows. However, to date, no country directly includes body energy status in national genetic evaluations. This is most likely due to the expense, difficulty, and therefore unfeasibility of collecting data on body energy status, particularly energy intake data, on sufficiently large numbers of animals. Methods of modeling body energy status without a requirement for data on feed intake across lactation have been proposed (Banos and Coffey, 2010). Such methods, however, require routine information on BCS and BW, which is still not feasible on a large number of commercial farms.

Recently, the mid-infrared (MIR) spectrum of milk was proposed as an indicator of body energy status in Holstein cows (McParland et al., 2011). Mid-infrared spectrometry is the method of choice worldwide for routine quantification of milk fat, protein, and lactose content of milk samples taken during milk testing. Thus, given the relatively high accuracy of prediction of energy status reported (up to $75 \%$; McParland et al., 2011), the MIR spectrum of milk could be a useful and routinely available cow management tool. However, the equations presented by McParland et al. 
(2011) to predict body energy status using milk MIR spectrometry were developed using data from a single research herd, albeit with 2 contrasting experimental dietary treatments differing in the level of concentrate offered. McParland et al. (2011) clearly showed that for equations to predict energy status accurately, the variation present in the validation data set must be representative of that in the calibration data set. The objective of the present study, therefore, was to further test the usefulness of the MIR spectrum of milk to predict body energy status across independent populations of indoor-fed versus grazing Holstein-Friesian dairy cows in the UK and Ireland, respectively.

\section{MATERIALS AND METHODS}

\section{Data Sets}

Two separate data sets were used in this study: (1) data from the Scottish Agricultural College Langhill dairy herd currently stationed at Crichton Royal Farm, Dumfries, Scotland (SAC); and (2) data from an Irish dairy research institute comprising 4 research herds, located at the Teagasc Animal and Grassland Research and Innovation Center, Moorepark, Co. Cork, Ireland (MPK).

The SAC data set comprised 1,218 cows from 2 genetically divergent lines of Holsteins, one control line selected to be the national average for milk fat plus protein yield, and the other line selected to be of high genetic merit for milk fat plus protein yield (Pryce et al., 1999). All SAC animals were divided into 2 dietary treatments: high concentrate (SAC-HC) and low concentrate (SAC-LC; Pryce et al., 1999). The MPK data set comprised 1,586 animals of differing strains of Holstein-Friesian (strains described by Coleman et al., 2010) on a predominantly grazed grass diet (diet described by Kennedy et al., 2008), with periodic concentrate supplementation.

\section{Production Data}

Performance data were available on 3,383 lactations from SAC and on 5,057 lactations from MPK between the years 1990 and 2010, inclusive. Before 2002, SAC cows were milked twice daily at 0400 and $1600 \mathrm{~h}$. From 2002 onward, milking was undertaken 3 times daily: $0415 \mathrm{~h}$ (SAC-AM), $1245 \mathrm{~h}$ (SAC-MD), and $1945 \mathrm{~h}$ (SAC-PM), and total daily and weekly average milk yields were recorded. Individual daily DMI was recorded during lactation using Calan gates (American Calan, Northwood, NH) for 3 successive days, followed by 3 $\mathrm{d}$ of no measurement. Body weight was recorded using automatic weighing scales 3 times per day following milking and averaged to a weekly BW value. Body condition score was assessed by the same operator weekly, using a scale of 0 to 5 with 0.25 -point intervals, where 0 is emaciated and 5 is obese (Lowman et al., 1976).

The MPK cows were milked twice daily at $0700 \mathrm{~h}$ (MPK-AM) and $1500 \mathrm{~h}$ (MPK-PM). Milk yield was recorded at each milking and summed to a daily milk yield. Body weight was recorded weekly on all MPK animals using a weighing scale following milking. Individual DMI was periodically recorded at grass using the $n$-alkane technique (Dillon and Stakelum, 1989) up to 12 times across lactation. Details on the technique are provided elsewhere (Kennedy et al., 2008). Body condition score was assessed weekly on a scale of 1 to 5 (Edmonson et al., 1989) by trained scorers and, in this study, was rescaled to the 0 to 5 BCS scale used on SAC cows.

In both research centers, milk fat and protein contents were recorded weekly. Age at calving was defined within parity and country as a class variable $(\mathrm{n}=3)$ with animals classed as (1) less than, (2) greater than, or (3) within 1 standard deviation of the median age at calving.

Two separate seasons of calving were defined for SAC and MPK animals. The SAC animals calve all year round; thus, the 2 seasons of calving were defined as February to August (inclusive) and September to January (inclusive). All MPK animals were spring calving; thus, season of calving was defined as January to February (inclusive) and March to June (inclusive).

\section{Computation of Body Energy Status}

Random regression models were fitted in ASReml (Gilmour et al., 2006), within parity, through the routine measures of milk yield, milk fat, and protein content, BW, and BCS. Data sets from each country were handled separately. Random regression models were fitted through routine measures of DMI in the SAC data set only. Random regression solutions were not generated for DMI of MPK animals because DMI was recorded only periodically on MPK animals throughout lactation. Thus, energy status, described later, was computed for MPK animals only during periods of lactation where true DMI was recorded.

The fitting of the random regression models to the SAC data was already described in detail by McParland et al. (2011), and a similar approach was taken when fitting random regression models to the MPK data. All random regression models included the fixed effects year of calving-by-season of calving, age at calving, year of record-by-month of record, and a fourthorder orthogonal polynomial on days postcalving, and the random effect of the interaction of cow by a 
fourth-order orthogonal polynomial on days postcalving. Random regression models fitted to the SAC data included the additional fixed effects of genetic line and feeding treatment. Random regression models fitted to the MPK data included the additional fixed effect of research herd $(\mathrm{n}=4)$. Six measurement error classes were fitted for days in milk $(4<$ DIM $\leq 10,10<$ $\mathrm{DIM} \leq 50,50<\mathrm{DIM} \leq 150,150<\mathrm{DIM} \leq 200,200$ $<$ DIM $\leq 250,250<$ DIM). Daily solutions from each random regression model were visually compared with the actual data collected for each trait and $10 \mathrm{MPK}$ and 19 SAC cow-lactations were discarded from the analysis because of poor concordance between the actual recorded data and the predicted values from the random regression models. All performance records from d 5 to 305 of lactation for animals in parities 1 to 4 were retained for analysis. Data on $811,888 \mathrm{SAC}$ test-days and 1,409,100 MPK test-days were available to compute body energy status.

Two separate measures of body energy status were computed for each day postcalving using the energy system outlined by Emmans (1994). The measures considered were (1) energy balance (Direct_EB; MJ/d), a function of milk yield, milk fat and protein contents, DMI, BW, and BCS; and (2) body energy content (EC; $\mathrm{MJ})$, a function of BW and BCS predicting body lipid and protein weight. These measures were previously described in detail (Banos and Coffey, 2010). Additional to Direct_EB and EC, the effective energy intake per day $(\mathbf{E E I}, \mathrm{MJ} / \mathrm{d})$ was computed as a function of $\mathrm{OM}$ intake and its digestible $\mathrm{CP}$ and $\mathrm{ME}$ content (Banos and Coffey, 2010).

\section{MIR Spectrum Data}

From June 2008 to August 2011, individual MPKAM and MPK-PM milk samples from all MPK animals were analyzed weekly using an MIR spectrometer (Foss MilkoScan FT6000, Hillerød, Denmark) and the resulting spectra were stored. The Foss MIR spectrum contains 1,060 data points that represent the absorption of infrared light through the milk sample at wavelengths in the 900 to $5,000 \mathrm{~cm}^{-1}$ region. Between September 2008 and December 2010, monthly milk samples from the SAC-AM, SAC-MD, and SAC-PM milkings on a given day for all SAC cows were sent to Teagasc Moorepark in Ireland for analysis using the same MIR spectrometer.

Only spectral data with an actual phenotypic record for all traits of milk yield, milk fat and protein contents, DMI, BW, and BCS within $14 \mathrm{~d}$ of the sampling date with MIR information were retained for analysis. These edits were undertaken to avoid any potential errors arising from extrapolation or interpolation between sampling dates with the random regressions. Following edits, 2,992 SAC-AM, 2,742 SAC-MD, and 2,989 SAC-PM spectral records from 564 lactations on 337 SAC cows were available. A subset of these data recorded between May 2008 and September 2010 were previously used by McParland et al. (2011) to predict energy status of Holsteins. An additional 844 MPK-AM and 820 MPK-PM spectral records from 338 lactations on $244 \mathrm{MPK}$ cows were available. Fewer records were available from the MPK cows because of the periodic recording of DMI in MPK. The average number of spectral records per lactation was 5.3, 4.9, and 5.3 for SAC-AM, SAC-MD, and SAC-PM milk samples, respectively, whereas the average number of spectral records per lactation was 2.5 and 2.4 for MPK-AM and MPK-PM milk samples, respectively.

\section{Development of Prediction Equations}

Partial least squares regression (Proc PLS; SAS Institute Inc., Cary, NC) was used to predict Direct_EB, EC, and EEI from the MIR linear absorbance data. Predictors included a subset of wavelengths in the 1,022 to $3,043 \mathrm{~cm}^{-1}$ range from the spectrum of 1,060 correlated wavelengths, together with milk yield; McParland et al. (2011) previously documented an increase in accuracy of prediction of body energy status when milk yield, which would be available at milk testing, was included in the prediction equation along with the MIR data.

Calibration and validation data sets were defined to develop and validate the prediction equations for Direct_EB, EC, and EEI, and the equations were developed using AM, MD (where available), and PM samples, separately. These equations were developed for use nationally, where, for the majority of milk samples, morning and evening samples are obtained and analyzed separately.

Six analyses were undertaken: (1) prediction equations were calibrated and externally validated within either the SAC or MPK research data set separately; (2) prediction equations were calibrated within the SAC data set and externally validated using the MPK data set; (3) the 2 research data sets were combined and the prediction equations calibrated and validated using the combined data set; (4) prediction equations were calibrated and validated using samples from early lactation (DIM <60) from the combined data set; (5) prediction equations were calibrated using samples from SAC-LC animals together with all MPK samples and were externally validated using samples from SAC$\mathrm{HC}$ cows; and (6) prediction equations were calibrated using samples from SAC-HC animals together with 
all MPK samples and were externally validated using samples from SAC-LC cows.

The mean bias of estimation, the slope between true and predicted values, the root mean square error (RMSE), and the accuracy (i.e., square root of the coefficient of multiple determinations - the correlation between the true and predicted energy status; $\mathbf{R}$ ) from the regression of true on predicted values of energy status were used to indicate the accuracy and robustness of each set of prediction equations obtained from both cross-validation and external validation. External validation was undertaken to test the usefulness of the prediction equations on data independent of the calibration data, thereby giving a truer reflection of the accuracy of prediction equations when used on a wider basis.

When the equations were calibrated and validated within the same research data set only (i.e., analysis 1 above), the data were stratified according to 3 criteria: feeding treatment, genetic line, and season of calving for SAC animals, and research herd, stage of lactation $(<100$ or $\geq 100$ DIM), and season of calving for MPK animals. Equations were calibrated using $75 \%$ of the data set within strata for each research data set and externally validated on the remaining $25 \%$. This procedure was iterated 4 times, each time using a different $25 \%$ of the data until all data were externally validated once. No animal was present in both the calibration and validation data sets in a given iteration. Accuracy of all equations was quantified using both split-sample crossvalidation and external validation on the 4 calibration and validation data sets, respectively. In addition, when the equations were developed within each research data set separately, the results provided represent the average number of records used in the external validation and the average accuracy of external validation and cross validation from 4 iterations of calibration and validation within research data set. When the equations were calibrated and validated within the same research data set, the bias and slope reported represent the largest absolute bias and the poorest slope (i.e., the greatest absolute difference from 1) from the 4 iterations of calibration and validation within the research data set.

Having the variation in the external validation data set represented in the calibration data set is necessary for a robust prediction equation (McParland et al., 2011). Therefore, in the present study, when equations were calibrated and externally validated using combined data from MPK and SAC animals, the data were sorted by each body energy status variable separately, and every fourth record was removed from the calibration data set for inclusion in the external validation data set.
Table 1. Mean performance (SD in parentheses) of body energy status and its component variables for animals from the Scottish research herd (SAC) and Irish (Moorepark) research herd (MPK) on days on which milk samples were analyzed using mid-infrared spectrometry

\begin{tabular}{lcc}
\hline Variable & SAC & MPK \\
\hline Cows (no.) & 337 & 245 \\
Records (no.) & 3,269 & 840 \\
Daily milk yield (kg) & $31.4(8.8)$ & $20.8(6.1)$ \\
Milk fat content (\%) & $3.8(0.7)$ & $4.1(0.6)$ \\
Milk protein content (\%) & $3.3(0.4)$ & $3.5(0.3)$ \\
Daily DMI (kg of DM/d) & $16.6(4.6)$ & $15.7(2.7)$ \\
BCS (units) & $2.1(0.3)$ & $2.3(0.3)$ \\
BW (kg) & $589.4(79.3)$ & $502.6(62.6)$ \\
Direct energy balance (MJ/d) & $-10.1(34)$ & $27.9(28)$ \\
Energy content (MJ) & $5,125(1,013)$ & $4,634(663)$ \\
Effective energy intake (MJ/d) & $168.0(46.7)$ & $158.9(26.7)$ \\
\hline
\end{tabular}

\section{RESULTS}

Mean performance of SAC and MPK animals for Direct_EB (MJ/d), EC (MJ), EEI (MJ/d), and component variables of Direct_EB including milk $(\mathrm{kg})$, milk fat and protein contents (\%), BCS (units), BW $(\mathrm{kg})$, and DMI ( $\mathrm{kg}$ of $\mathrm{DM} / \mathrm{d})$ are presented in Table 1 for days coinciding with when MIR spectral data were available. The MPK cows had a lower milk yield but higher milk constituents than the SAC cows. The MPK animals also had lower BW than the SAC cows yet had slightly better BCS. On average, MPK cows were in greater Direct_EB than the SAC cows, despite the lower DMI of the MPK cows (Table 1, Figure 1).

Summary statistics for the predictive ability of the equations developed are provided in Tables 2, 3, and 4 for when calibration and validation were undertaken within each research data set (Table 2), across research data sets (Table 3), and from the combined data sets (Table 4), respectively. Summary statistics from the calibration and validation of prediction equations in different dietary treatments are provided in Tables 5 and 6 . For the purposes of this study, accuracy of prediction $(\mathrm{R})$ was defined as the square root of the coefficient of determination from the regression model of true on predicted values of body energy status. Unless otherwise stated, the reported accuracy of the prediction equations is based on external validation.

\section{Prediction Equations Calibrated and Validated Within the Same Research Data Set}

When the equations were calibrated and validated within the same research data set, the bias and slope reported represent the largest absolute bias and the poorest slope (i.e., greatest absolute difference from 1) from the 4 iterations of calibration and validation within research data set. Accuracy of prediction of Direct_EB 


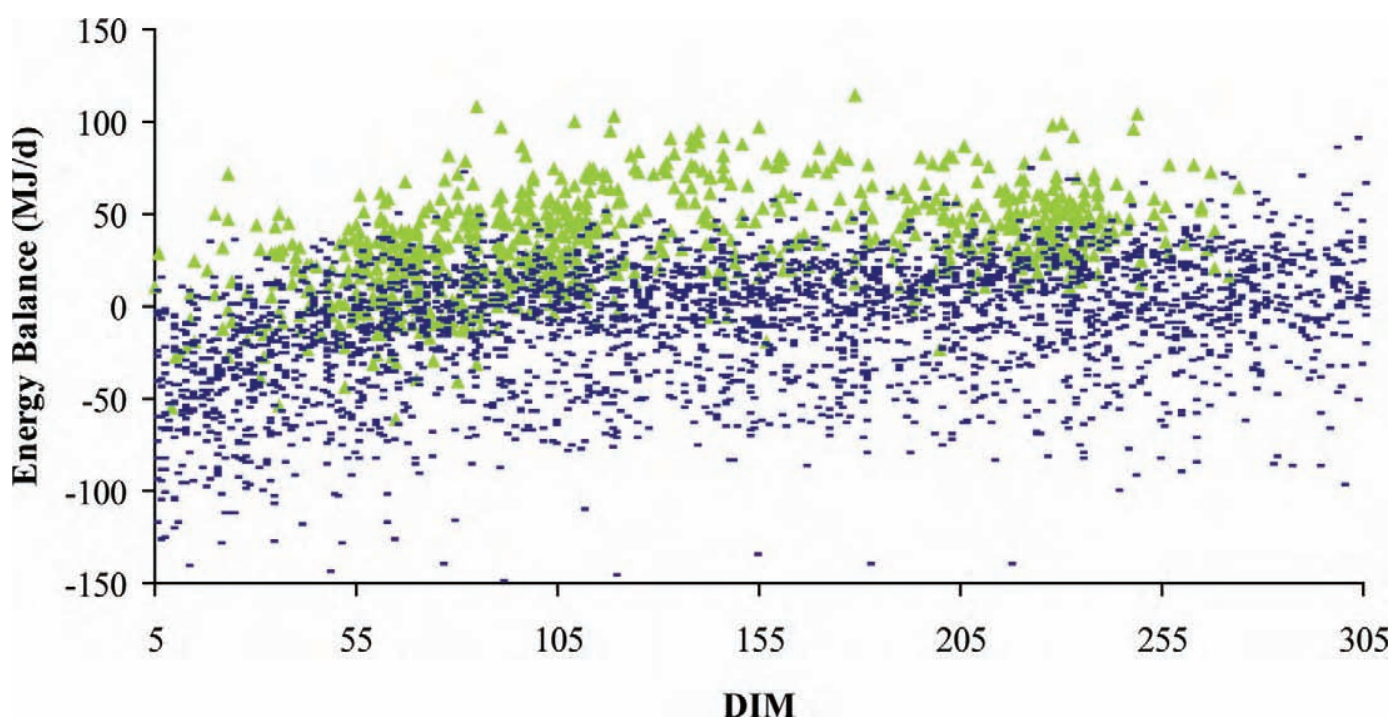

Figure 1. Calculated energy balance for the Scottish research herd (-) and Irish research herd (gray triangles) animals. Color version available in the online PDF.

ranged from 0.65 (SAC-PM) to 0.69 (SAC-MD) when equations were both calibrated and validated within either the SAC data set or within the MPK data set separately (Table 2). Generally, the average bias was closer to zero than the maximum absolute bias reported in Table 2. Although the maximum bias is presented (Table 2), the bias from $80 \%$ of the iterations was not different $(P>0.05)$ from zero. Similarly, the slope of true on predicted values of Direct_EB across iterations was generally much closer to 1 than the poorest slope reported in Table 2; 50\% of the regression coefficients were not different $(P>0.05)$ from 1.

Unlike Direct_EB, the average accuracy of the EC prediction equations calibrated and validated using MPK samples was considerably poorer than corresponding equations calibrated and validated in the

Table 2. Within-herd calibration and validation data sets, root mean square error (RMSE), correlation coefficient (R), number of records in external validation (Recs), maximum bias $^{1}$ and slope $^{2}$ (b) obtained from predicting direct energy balance (Direct_EB), energy content, and effective energy intake, and tested using split-sample cross-validation and external validation methods

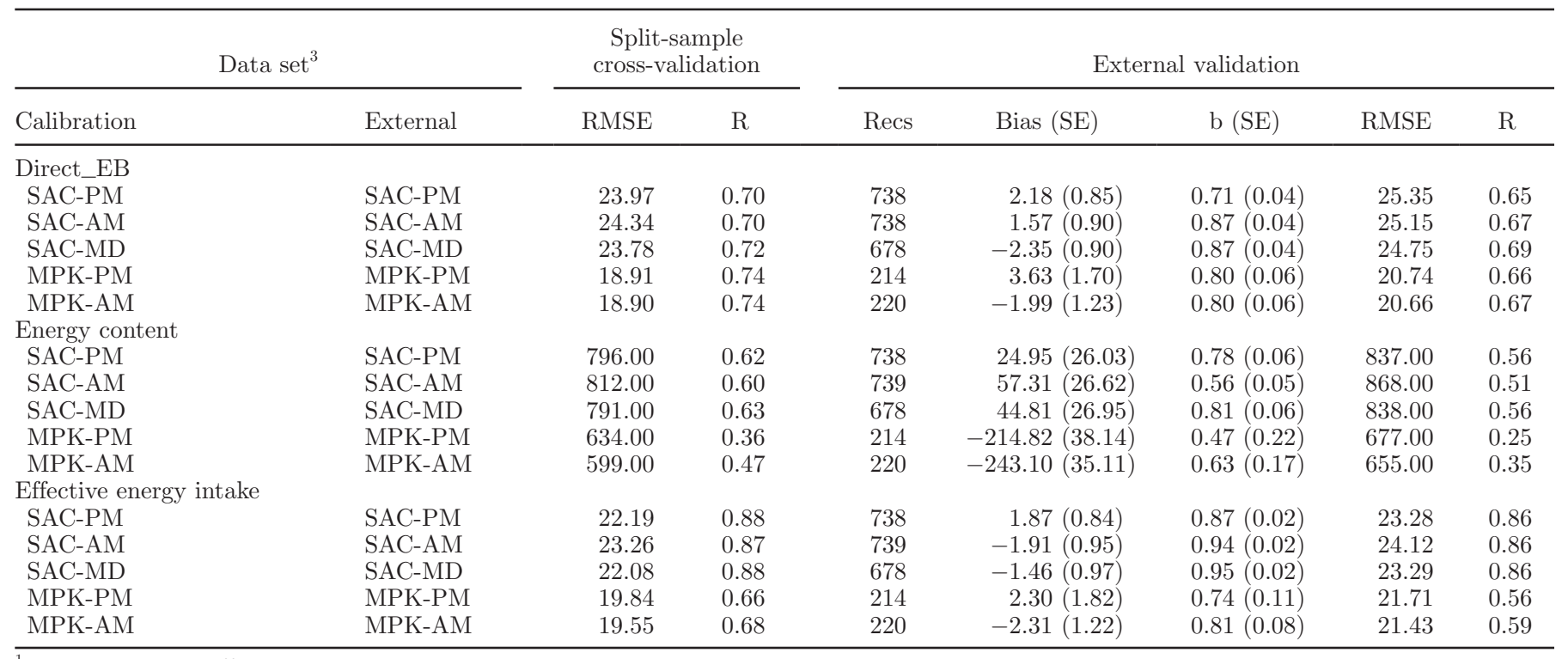

${ }^{1}$ Largest average difference between predicted values and true values in any external validation data set.

${ }^{2}$ Linear regression coefficient of true energy status value on predicted value.

${ }^{3} \mathrm{SAC}=$ Scottish research herd; MPK $=$ Irish (Moorepark) research herd; AM = morning milk; $\mathrm{MD}=$ midday milk; PM = evening milk. 
Table 3. Across-herd calibration and validation data sets, root mean square error (RMSE), correlation coefficient (R), number of records in external validation (Recs), bias, and slope (b) obtained from predicting direct energy balance (Direct_EB), energy content, and effective energy intake, and tested using split-sample cross-validation and external validation methods

\begin{tabular}{|c|c|c|c|c|c|c|c|c|}
\hline \multicolumn{2}{|c|}{ Data set $^{1}$} & \multicolumn{2}{|c|}{$\begin{array}{l}\text { Split-sample } \\
\text { cross-validation }\end{array}$} & \multicolumn{5}{|c|}{ External validation } \\
\hline Calibration & External & RMSE & $\mathrm{R}$ & Recs & Bias (SE) & b (SE) & RMSE & $\mathrm{R}$ \\
\hline \multicolumn{9}{|l|}{ Direct_EB } \\
\hline SAC-PM & MPK-PM & 23.74 & 0.70 & 837 & $62.84(1.19)$ & $0.11(0.04)$ & 27.77 & 0.09 \\
\hline SAC-AM & MPK-PM & 24.50 & 0.69 & 837 & $69.92(1.32)$ & $0.08(0.03)$ & 27.79 & 0.09 \\
\hline SAC-MD & MPK-PM & 24.01 & 0.71 & 837 & $70.49(1.32)$ & $0.14(0.03)$ & 27.58 & 0.15 \\
\hline SAC-MD & MPK-AM & 24.01 & 0.71 & 862 & $45.52(1.21)$ & $0.08(0.04)$ & 28.00 & 0.07 \\
\hline \multicolumn{9}{|c|}{ Energy content } \\
\hline SAC-PM & MPK-PM & 793.08 & 0.62 & 837 & $-469.75(30.72)$ & $0.14(0.04)$ & 680.57 & 0.13 \\
\hline SAC-AM & MPK-PM & 828.00 & 0.58 & 837 & $-776.05(34.54)$ & $0.06(0.03)$ & 685.12 & 0.07 \\
\hline SAC-MD & MPK-PM & 794.46 & 0.62 & 837 & $-602.97(34.88)$ & $0.08(0.03)$ & 683.81 & 0.09 \\
\hline SAC-PM & MPK-AM & 793.08 & 0.62 & 862 & $-402.69(28.64)$ & $0.14(0.04)$ & 679.79 & 0.12 \\
\hline SAC-AM & MPK-AM & 828.00 & 0.58 & 862 & $-643.41(30.01)$ & $0.07(0.04)$ & 683.66 & 0.06 \\
\hline SAC-PM & MPK-AM & 23.03 & 0.87 & 862 & $33.29(1.25)$ & $0.18(0.03)$ & 25.96 & 0.21 \\
\hline SAC-AM & MPK-AM & 23.40 & 0.87 & 862 & $37.12(1.33)$ & $0.17(0.02)$ & 25.84 & 0.23 \\
\hline SAC-MD & MPK-AM & 22.23 & 0.88 & 862 & $31.50(1.28)$ & $0.21(0.03)$ & 25.58 & 0.27 \\
\hline
\end{tabular}

${ }^{1} \mathrm{SAC}=$ Scottish research herd; MPK = Irish (Moorepark) research herd; AM = morning milk; MD = midday milk; PM = evening milk.

SAC data set (Table 2). The mean bias between true and predicted values was not different from zero for any iterations using the SAC data, yet bias exceeded 100 MJ for $50 \%$ of iterations in the MPK data set. However, the average RMSE was higher for SAC equations compared with MPK equations, reflecting the greater variance of EC in the SAC animals (Table 2).

Accuracy of prediction of EEI within the SAC data set was good $(R=0.86$; Table 2$)$ but was poorer $(R$ $=0.56-0.59)$ when equations were calibrated and validated using only the MPK data set (Table 2). However, as for EC, the prediction equations for EEI developed using MPK samples had a lower average RMSE than the equations developed using SAC samples, yet had a larger mean bias and poorer slope between true and predicted values (Table 2). The slope between true and predicted values was different from 1 in $63 \%$ of iterations across the MPK data set compared with $25 \%$ of iterations across the SAC data set. The mean bias was generally not different from zero across iterations of SAC or MPK data sets; however, the mean standard error across iterations was lower for the SAC data set (0.9 MJ) than it was for the MPK data set (1.5 MJ).

\section{Prediction Equations Calibrated and Validated in Different Research Data Sets}

Despite the high accuracy of cross-validation, equations developed using SAC data were not useful to pre- dict Direct_EB, EC, or EEI in MPK animals (Table 3). The slope between true and predicted values was close to zero, and large mean biases were associated with the energy status predictions (Table 3 ).

\section{Prediction Equations Calibrated and Validated in Combined Research Data Sets}

The accuracy of predicting Direct_EB and EC from records across the entire lactation, using the 2 research data sets combined to calibrate and externally validate equations (Table 4), was similar to the accuracy of the prediction equations calibrated and validated within herd (Table 2). However, the accuracy of predicting EEI was slightly poorer than the accuracy of predicting EEI within the SAC herd (Tables 2 and 4). The greatest variation in prediction accuracy across different milk samples used was in the prediction of Direct_EB, where accuracy of external validation ranged from 0.47 (SAC-PM and MPK-AM samples) to 0.69 (SAC-MD and MPK-AM samples). In contrast, accuracy of validation of EC ranged from 0.51 to 0.56 and that of EEI ranged from 0.76 to 0.80 .

When prediction equations were calibrated and validated using samples from early lactation (i.e., DIM $<60$ ), accuracy of prediction of Direct_EB was slightly poorer than that across the entire lactation, but the accuracy of prediction of both EC and EEI was slightly improved (Table 4). 
Table 4. Combined research herd calibration and validation data sets in the entire lactation and in early lactation, root mean square error (RMSE), correlation coefficient (R), number of records in external validation (Recs), bias, and slope (b) obtained from predicting direct energy balance (Direct_EB), energy content, and effective energy intake, and tested using split-sample cross-validation and external validation methods

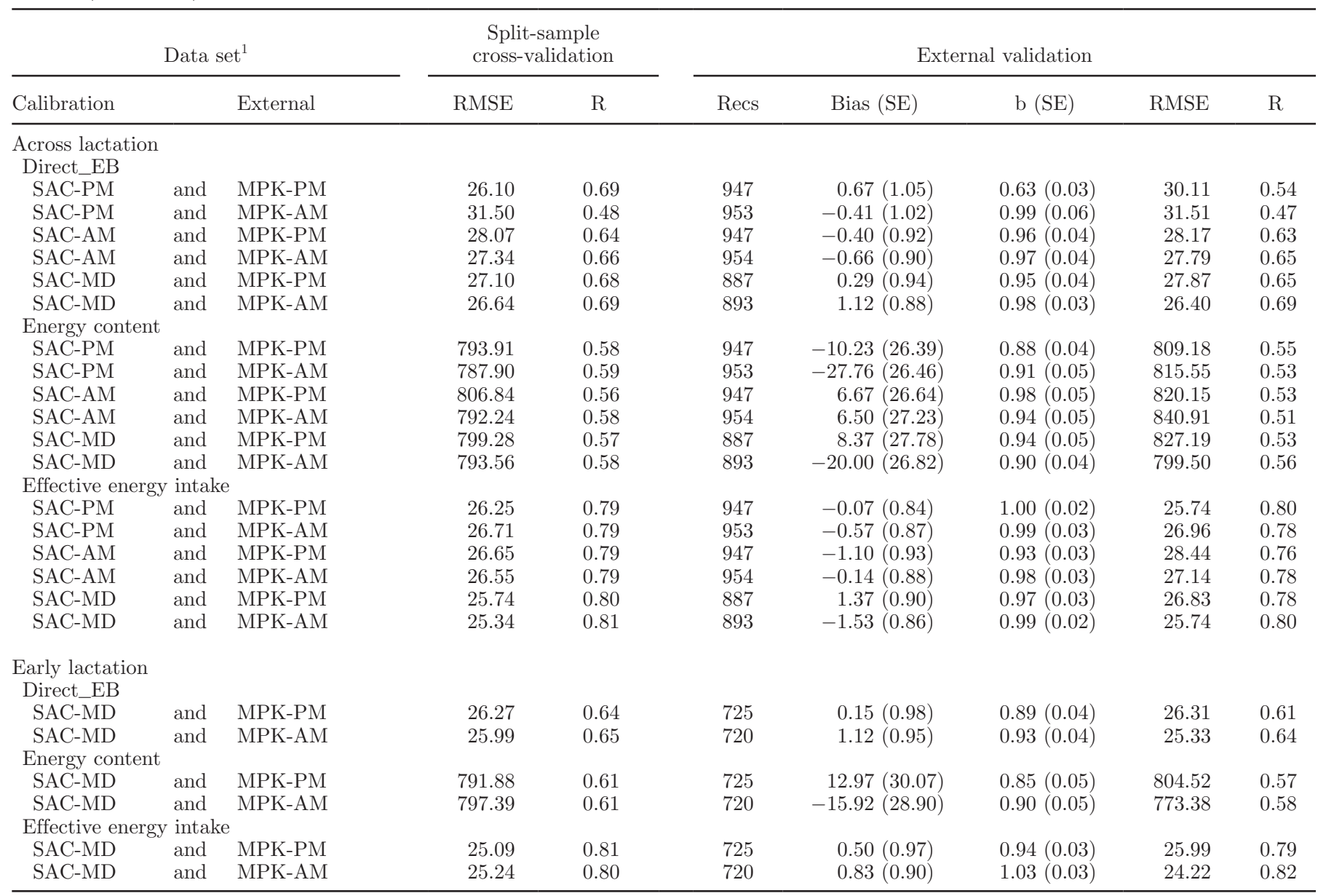

${ }^{1} \mathrm{SAC}=$ Scottish research herd; MPK $=$ Irish (Moorepark) research herd; AM = morning milk; $\mathrm{MD}=$ midday milk; PM = evening milk.

\section{Prediction Equations Calibrated and Validated in Different Feed Systems}

Accuracy of prediction of body energy status was poorer than that obtained from the combined data set when equations were calibrated using samples from MPK and one SAC feeding treatment and validated on SAC animals on the other feeding treatment (Tables 5 and 6). Accuracy of validation of Direct_EB ranged from 0.28 to 0.46 (Table 5) when equations were validated on samples from cows on the high concentrate diet; the range in accuracy of predicting Direct_EB for cows on the low concentrate diet varied from 0.21 to 0.33 (Table 6). The slope between true and predicted values of Direct_EB was poor (0.30-0.52) when equations were validated on samples from cows fed the high concentrate diet (Table 5); the slope between true and predicted values of Direct_EB was $\leq 0.69$ when equations were validated on samples from cows on the low concentrate diet (Table 6).
Accuracy of prediction of both EC and EEI was lower than the accuracy obtained from the full combined data set when equations were calibrated using data from MPK and one SAC feeding treatment only. The slope between true and predicted values of EEI was generally $>1$ when equations were validated on samples from cows on the low concentrate diet (Table 6).

\section{DISCUSSION}

The importance of energy status on dairy cow performance is well accepted (Beam and Butler, 1999). Therefore, data on cow or herd average energy status could be very useful in aiding cow or herd management but also in breeding programs to increase the accuracy of selection, especially for low heritability traits such as health (Berry et al., 2011) or fertility (Pryce and Veerkamp, 2001). Nonetheless, low-cost measures of predicting energy balance are currently not 
Table 5. Solutions from equations calibrated using samples from SAC low-concentrate-fed animals and MPK animals and validated on SAC high-concentrate-fed animals, root mean square error (RMSE), correlation coefficient (R), number of records in external validation (Recs), bias, and slope (b) obtained from predicting direct energy balance (Direct_EB), energy content, and effective energy intake, and tested using splitsample cross-validation and external validation methods

\begin{tabular}{|c|c|c|c|c|c|c|c|}
\hline \multirow{2}{*}{$\frac{\text { Data set }^{1}}{\text { Calibration and external }}$} & \multicolumn{2}{|c|}{$\begin{array}{l}\text { Split-sample } \\
\text { cross-validation }\end{array}$} & \multicolumn{5}{|c|}{ External validation } \\
\hline & RMSE & $\mathrm{R}$ & Recs & $\operatorname{Bias}(\mathrm{SE})$ & b (SE) & RMSE & $\mathrm{R}$ \\
\hline \multicolumn{8}{|l|}{ Direct_EB } \\
\hline SAC-PM and MPK-PM & 22.88 & 0.75 & 1,498 & $23.11(0.73)$ & $0.40(0.03)$ & 24.55 & 0.36 \\
\hline SAC-PM and MPK-AM & 21.62 & 0.78 & 1,498 & $19.41(0.67)$ & $0.52(0.03)$ & 23.47 & 0.46 \\
\hline SAC-AM and MPK-PM & 23.37 & 0.76 & 1,497 & $22.36(0.80)$ & $0.30(0.03)$ & 25.38 & 0.28 \\
\hline SAC-MD and MPK-AM & 22.04 & 0.79 & 1,381 & $21.56(0.78)$ & $0.37(0.03)$ & 24.74 & 0.35 \\
\hline \multicolumn{8}{|l|}{ Energy content } \\
\hline SAC-PM and MPK-PM & 727.66 & 0.60 & 1,498 & $107.91(24.14)$ & $0.72(0.04)$ & 921.70 & 0.39 \\
\hline SAC-PM and MPK-AM & 728.18 & 0.60 & 1,498 & $93.33(23.98)$ & $0.74(0.04)$ & 917.59 & 0.40 \\
\hline SAC-AM and MPK-PM & 723.77 & 0.61 & 1,497 & $191.76(24.22)$ & $0.71(0.04)$ & 924.04 & 0.39 \\
\hline SAC-AM and MPK-AM & 746.17 & 0.57 & 1,497 & $204.05(25.34)$ & $0.58(0.05)$ & 953.61 & 0.31 \\
\hline SAC-MD and MPK-PM & 748.89 & 0.56 & 1,381 & $267.35(26.31)$ & $0.63(0.05)$ & 960.27 & 0.31 \\
\hline SAC-AM and MPK-AM & 22.77 & 0.83 & 1,497 & $21.13(0.71)$ & $0.68(0.02)$ & 25.89 & 0.61 \\
\hline SAC-MD and MPK-PM & 22.06 & 0.84 & 1,381 & $19.21(0.73)$ & $0.68(0.02)$ & 25.57 & 0.60 \\
\hline SAC-MD and MPK-AM & 21.78 & 0.84 & 1,381 & $18.83(0.70)$ & $0.71(0.02)$ & 24.87 & 0.63 \\
\hline
\end{tabular}

${ }^{1} \mathrm{SAC}=$ Scottish research herd; MPK $=$ Irish (Moorepark) research herd; AM = morning milk; $\mathrm{MD}=$ midday milk; PM = evening milk.

Table 6. Solutions from equations calibrated using samples from SAC high-concentrate-fed animals and MPK animals and validated on SAC low-concentrate-fed animals, root mean square error (RMSE), correlation coefficient (R), number of records in external validation (Recs), bias, and slope (b) obtained from predicting direct energy balance (Direct_EB), energy content, and effective energy intake, and tested using splitsample cross-validation and external validation methods

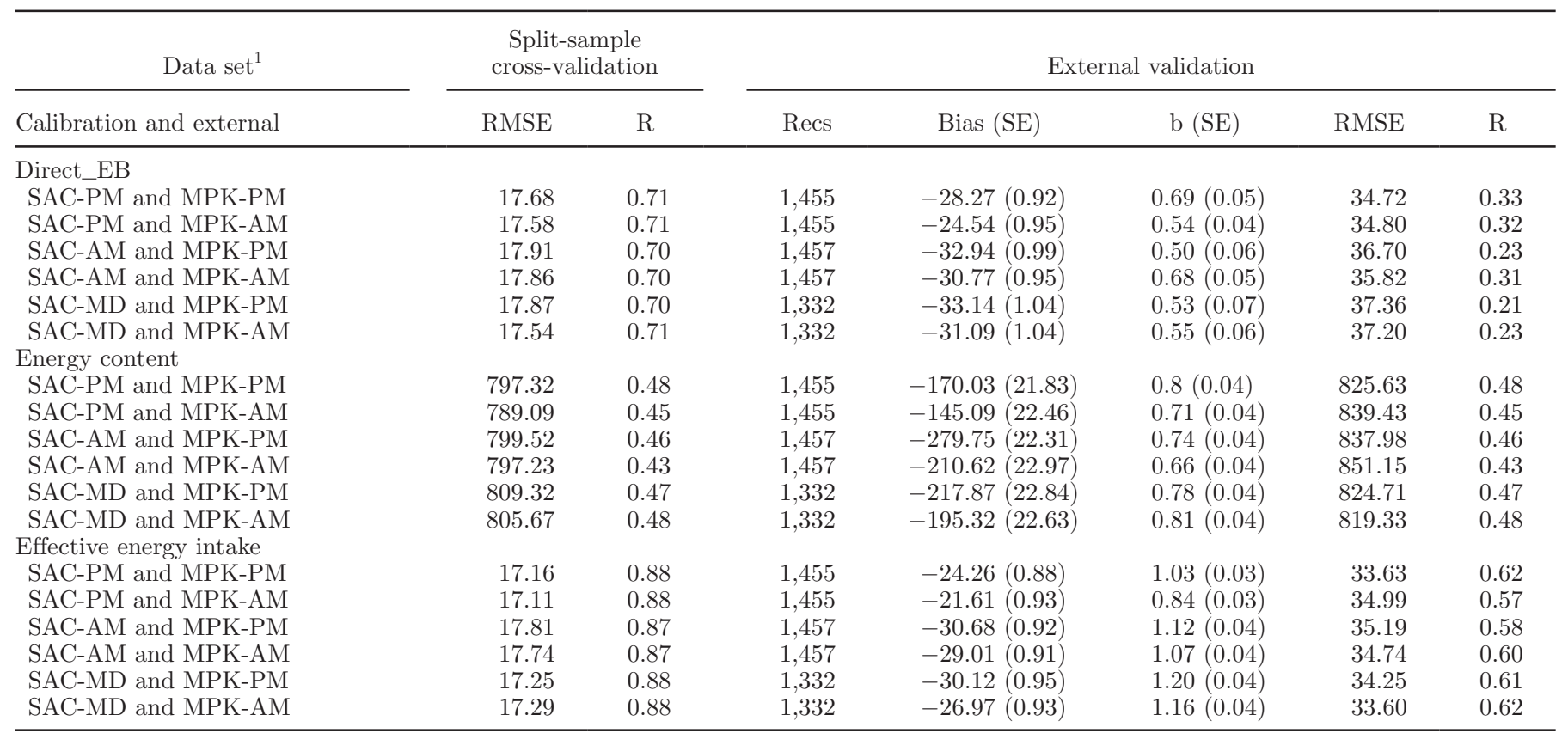

${ }^{1} \mathrm{SAC}=$ Scottish research herd; MPK $=$ Irish (Moorepark) research herd; AM = morning milk; MD = midday milk; PM = evening milk. 
available. McParland et al. (2011) hypothesized that because mid-infrared spectrometry is the method of choice worldwide for routine quantification of milk fat, protein, and lactose contents, and now milk fatty acid composition (Soyeurt et al., 2011) in milk samples, and given the strong association between these variables and energy balance (Buttchereit et al., 2011), direct prediction of energy balance from the MIR spectrum of milk should reduce prediction errors. McParland et al. (2011) proposed their energy status prediction equations from milk MIR spectra as a possible useful tool in cow or herd management because (1) relatively good accuracies of prediction were reported, and (2) MIR data are generated for all cows at the time of milk recording as well as for herd bulk milk tank samples and therefore no additional sampling would be required for energy status predictions to be generated on a routine basis. However, McParland et al. (2011) clearly showed that the variation in the population within which the developed equations were to be exploited should be represented in the data set used to calibrate the initial prediction equations. Furthermore, McParland et al. (2011) used data from only one research herd, albeit with 2 feeding systems, both of which involved indoor feeding at the time of measurement.

The objective of this study was to further develop the principle reported by McParland et al. (2011) that the MIR spectrum of milk could be useful to predict cow energy status using data from 2 diverse production sys- tems as well as genetic groups of differing ancestry. The SAC research herd comprises 2 lines of North American Holsteins divergent for genetic merit split across 2 TMR diets ( 1 high concentrate and 1 low concentrate). In contrast, samples used from the MPK research herd mainly included New Zealand Holstein-Friesians, which were offered a predominantly grazed grass diet with periodic concentrate supplementation. These differences in research populations used, one based in Scotland and one in Ireland, was reflected in differences in performance (Table 1, Figure 1) as well as in different milk spectral profiles (Figure 2).

\section{Predictive Ability}

Substantiating the study of McParland et al. (2011), the variation in energy status (and therefore MIR spectra) in the population where the prediction equations are going to be exploited needs to be represented in the data set used to calibrate the equations. This was evident when prediction equations were calibrated in SAC cows and applied to MPK cows. The MPK and SAC research herds differed in mean levels of individual component variables of energy status (Table 1), measurement techniques of the component variable DMI, and actual energy status levels. Differences in Direct_ EB, EC, and EEI of the 2 research data sets (Table 1, Figure 1), combined with differences in the spectral data of the 2 herds (Figure 2), resulted in equations

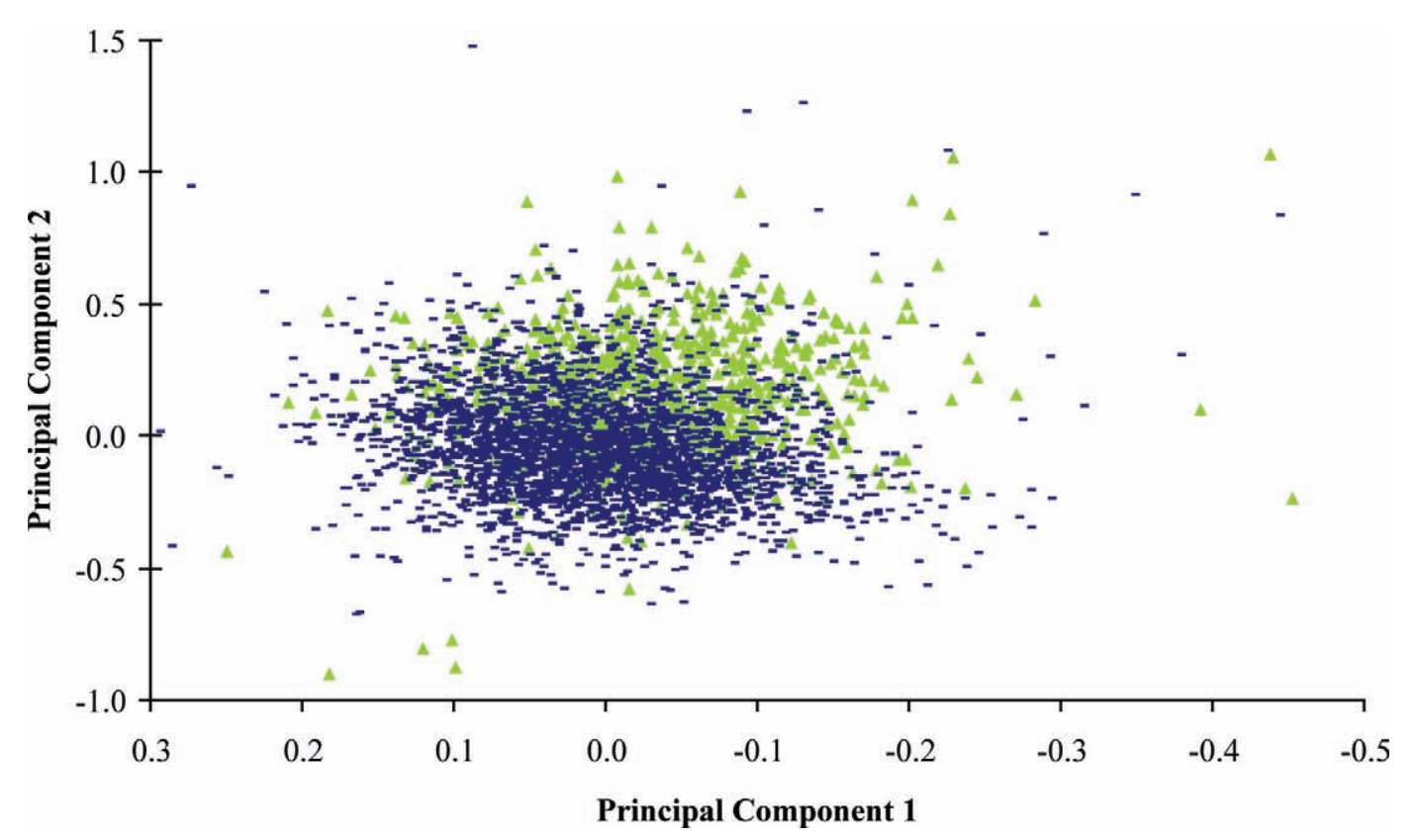

Figure 2. First 2 principal components of spectral data of milk from the Scottish research herd (-) and Irish research herd (gray triangles) animals. Color version available in the online PDF. 
calibrated using phenotypic and spectral data that did not represent the predicted data set. Thus, equations were unable to "recognize" either the body energy status profiles or the spectral data of the validation data set.

The Direct_EB profile of the SAC-HC animals was more similar to that of MPK animals than to the SACLC Direct_EB profiles (data not shown). Thus, when equations were calibrated using samples from SAC-LC plus MPK animals and validated using milk samples from SAC-HC animals, the accuracy of prediction of Direct_EB was greater and the mean prediction bias was smaller than when equations were developed using SAC-HC plus MPK and validated on SAC-LC (Tables 5 and 6). The inclusion of MPK animals in the calibration of the equations may have been useful to aid prediction of SAC-HC animals. However, the prediction accuracy obtained across feed system was poor.

Corroborating the results from this study, McParland et al. (2011), using a subset of the SAC data used in the present study, reported that the equations to predict body energy status were generally most accurate at predicting EEI and least accurate at predicting EC. The generally greater accuracy of prediction of Direct_EB relative to EC in both the present study and the study of McParland et al. (2011) may be a reflection of the strong accuracy of prediction for some of the component variables of Direct_EB using the MIR spectrum. Direct_EB comprises milk fat, protein, and lactose contents, all of which are routinely predicted using the MIR spectrum, as well as milk yield, which was included in the prediction equation for energy status in the present study. Direct_EB also incorporates EEI, which was accurately predicted from the MIR spectrum (Tables 2 to 5). Energy content, on the other hand, was calculated from BCS and BW, neither of which is routinely predicted using the MIR spectrum; McParland et al. (2011) previously reported poor accuracy of prediction of BCS from the milk MIR spectrum. Furthermore, BCS is subjectively scored and BW (change) is subject to random error such as gut fill. The DMI reported for the SAC herd is measured differently from the DMI reported for the MPK herds. However, EC does not consider DMI in its calculation, and thus could have been a better trait for prediction across countries.

It is unclear why the prediction accuracies of equations developed using the MPK data sets were generally lower than those developed using the SAC data set. Although an accurate measurement of DMI, the n-alkane method to predict the DMI of the MPK herd might have been less accurate than the Calan gate method of assessing DMI used in the SAC herd, and this had a greater effect on the predictive ability of EEI than on Direct_EB. Considerably more data were available from SAC to develop the prediction equations, which is also likely to influence accuracy of prediction.

\section{Implications}

Equations to predict body energy status have been developed using a joint data set obtained from research herds in Ireland and Scotland. The equations to predict Direct_EB are sufficiently accurate to provide useful information to dairy farmers regarding the energy status levels of their herd. Because these equations require only information from the MIR spectrum, which is routinely generated during milk analyses, and information on milk yield, information on the energy status levels of herds can now be provided to farmers. Farmers who are actively milk recording can also receive information on the individual energy status profiles of cows at the time of milk recording at no additional cost. However, it was clear from this study, corroborating the recommendations from a previous study on energy status prediction (McParland et al., 2011), that the variation present in the population in which any prediction equations are to be exploited must be appropriately represented in the calibration data set. Because individual animal energy status will not be available in commercial populations, the MIR spectra of the animals can be compared with that in the calibration data set (Figure 2) and a decision made on whether the patterns in the spectra are represented in the calibration data set. However, a more obvious solution would be to collate data on a sufficiently large population of animals from diverse breeds, feed systems, and energy status, and to develop the equations using these data. This can be best achieved through international collaboration.

Through the routine collation of MIR-predicted energy status, longitudinal herd (and cow) data can subsequently be used to monitor changes in energy status of the herd over time and therefore evaluate, in real time, the effect of changes in management practice on herd energy status. Adjustment for fixed effects such as herd parity structure and genetic merit can facilitate benchmarking of individual herds against contemporaries. Knowledge on energy balance status, but probably more importantly, the change in herd average energy status, can provide valuable information for inclusion in farm decision-support tools.

Although every attempt was made in this study to minimize the influence of random noise in the phenotypic values of the component traits of energy balance, inaccuracies in the collection of these data, especially feed intake and BW, are likely to exist. Therefore, the expected accuracy of prediction of energy status, as 
defined in this study, was not expected to be very high. The expected random noise present may be minimized further, although not alleviated, by more accurate measurement of energy balance in calorimetric chambers.

Genetic gain in low-heritability traits such as health and fertility is hampered by relatively low accuracy of selection. Although accuracy of selection can be improved through greater levels of recording among other things, accuracy can also be increased by including heritable, genetically correlated traits in multi-trait genetic evaluations for health and fertility. Although no variance components were estimated in the present study for energy balance, previous studies (Berry et al., 2007) documented significant genetic variation in energy status. However, precise estimates of the genetic correlations between energy status and either health or fertility traits are lacking, predominantly because of a lack of sufficiently large data sets with phenotypes for energy status. Access to MIR-predicted energy status could overcome this limitation because MIR spectra should be available from all milk samples taken at milk recording. If the heritability of predicted energy status is similar to that of milk yield, as previously suggested (Berry et al., 2007), the accuracy of genetic evaluations for energy status would be similar to that of genetic evaluations for milk. Currently, $49 \%$ of cows in Ireland are milk recorded between 4 and 12 times during lactation, and at least $50 \%$ of cows in the UK are milk recorded approximately 10 times during lactation.

\section{ACKNOWLEDGMENTS}

The RobustMilk project is financially supported by the European Commission under the Seventh Framework Programme, Grant Agreement KBBE-211708. The content of this paper is the sole responsibility of the authors, and it does not necessarily represent the views of the Commission or its services. The authors gratefully acknowledge the work by staff at Teagasc Moorepark and at Crichton Research Farm and the assistance of Anne Geoghegan (Teagasc Moorepark) and Ross McGinn (SAC, Midlothian, UK) in collating the phenotypic data used in this study.

\section{REFERENCES}

Banos, G., and M. P. Coffey. 2010. Genetic association between body energy measured throughout lactation and fertility in dairy cattle. Animal 4:189-199.

Beam, S. W., and W. R. Butler. 1999. Effects of energy balance on follicular development and first ovulation in postpartum dairy cows. J. Reprod. Fertil. Suppl. 54:411-424.

Berry, D. P., M. L. Bermingham, M. Good, and S. J. More. 2011. Genetics of animal health and disease in cattle. Ir. Vet. J. 64:5.

Berry, D. P., B. Horan, M. O'Donovan, F. Buckley, E. Kennedy, M. McEvoy, and P. Dillon. 2007. Genetics of grass dry matter intake, energy balance and digestibility in Irish dairy cows. J. Dairy Sci. 90:4835-4845.

Buttchereit, N., E. Stamer, W. Junge, and G. Thaller. 2011. Short communication: Genetic relationships among daily energy balance, feed intake, body condition score, and fat to protein ratio of milk in dairy cows. J. Dairy Sci. 94:1586-1591.

Coleman, J., D. P. Berry, K. M. Pierce, A. Brennan, and B. Horan. 2010. Dry matter intake and feed efficiency profiles of 3 genotypes of Holstein-Friesian within pasture-based systems of milk production. J. Dairy Sci. 93:4318-4331.

Dillon, P. G., D. P. Berry, R. D. Evans, F. Buckley, and B. Horan. 2006. Consequences of genetic selection for increased milk production in European seasonal pasture based systems of milk production. Livest. Prod. Sci. 99:141-158.

Dillon, P. G., and G. Stakelum. 1989. Herbage and dosed alkanes as a grass management technique for dairy cows. Ir. J. Agric. Res. 8:104. (Abstr.)

Edmonson, A. J., I. J. Lean, L. D. Weaver, T. Farver, and G. Webster. 1989. A body condition scoring chart for Holstein dairy cows. J. Dairy Sci. 72:68-78.

Emmans, G. C. 1994. Effective energy: A concept of energy utilization applied across species. Br. J. Nutr. 71:801-821.

Gilmour, A. R., B. J. Gogel, B. R. Cullis, and R. Thompson. 2006. ASReml User Guide, Release 2.0. VSN International Ltd., Hemel Hempstead, UK.

Kennedy, E., M. O'Donovan, L. Delaby, and F. P. O’Mara. 2008. Effect of herbage allowance and concentrate supplementation on dry matter intake, milk production and energy balance of early lactating dairy cows. Livest. Sci. 117:275-286.

Lowman, B. G., N. Scott, and S. Somerville. 1976. Condition Scoring of Cattle. Rev. ed. Bulletin of the East Scotland College of Agriculture, No. 6. ESCA, Edinburgh, UK

McParland, S., G. Banos, E. Wall, M. P. Coffey, H. Soyeurt, R. F. Veerkamp, and D. P. Berry. 2011. The use of mid-infrared spectrometry to predict body energy status of Holstein cows. J. Dairy Sci. 94:3651-3661.

Pryce, J. E., B. L. Nielsen, R. F. Veerkamp, and G. Simm. 1999 Genotype and feeding system effects and interactions for health and fertility traits in dairy cattle. Livest. Prod. Sci. 57:193-201.

Pryce, J. E., and R.F. Veerkamp. 2001 The incorporation of fertility indices in genetic improvement programmes. Pages 237-249 in Proc. Fertility in the High Producing Dairy Cow, Ireland. Br. Soc. Anim. Sci. Occ. Publ. 26. Br. Soc. Anim. Sci. Penicuik, UK.

Soyeurt, H., F. Dehareng, N. Gengler, S. McParland, E. Wall, D. P. Berry, M. Coffey, and P. Dardenne. 2011. Mid-infrared prediction of bovine milk fatty acids across multiple breeds, production systems, and countries. J. Dairy Sci. 94:1657-1667. 